\title{
Immunomodulatory Therapy with Glatiramer Acetate Reduces Endoplasmic Reticulum Stress and Mitochondrial Dysfunction in Experimental Autoimmune Encephalomyelitis
}

Tapas K. Makar ${ }^{1,2}$, Poornachander R Guda ${ }^{1}$, Sugata Ray ${ }^{1}$, Sanketh Andhavarapu ${ }^{1}$, Vamshi KC Nimmagadda ${ }^{1}$, Volodymyr Gerzanich ${ }^{2}$, Marc J Simard ${ }^{1,2}$, and Christopher T. Bever, Jr.1,3

(1) Research Service, VA Maryland Health Care system, Baltimore, MD

(2) Department of Neurosurgery, University of Maryland, School of Medicine

(3) Office of Research and Development, Department of Veterans Affairs

Corresponding author: Tapas Kumar Makar (ORCID ID: 0000-0002-1171-061X), Institute of Human Virology, 725 W Lombard St, Baltimore, MD 21201. Email:

tmakar@ihv.umaryland.edu, Telephone: 443-527-0149 


\title{
Running Title: GA reduces ER \& mitochondrial stress in EAE
}

\begin{abstract}
Endoplasmic reticulum (ER) stress and mitochondrial dysfunction are found in lesions of multiple sclerosis (MS) and of experimental autoimmune encephalomyelitis (EAE), an animal model of MS, and may contribute to the neuronal loss that underlies permanent impairment. These pathological changes are due to the neuroinflammation that characterizes MS and the strong interplay between the ER and mitochondria. We investigated whether immunomodulatory drug glatiramer acetate (GA) can reduce these changes in the spinal cords of chronic EAE mice by using routine histology, immunostaining, and electron microscopy. EAE spinal cord tissue exhibited increased infiltration/inflammation (upregulation of proinflammatory cytokines), demyelination, mitochondrial dysfunction (increased fission, decreased fusion, and increased biogenesis), ER stress, downregulation of NAD+ dependent pathways, and increased neuronal death. GA reversed these pathological changes, suggesting that immunomodulating therapy can attenuate ER stress, mitochondrial dysfunction, apoptotic cell death, and demyelination in the CNS and increase NAD+ utilizing activities by suppressing neuroinflammation.
\end{abstract}

Key words: Endoplasmic reticulum stress, mitochondrial dysfunction, multiple sclerosis, experimental autoimmune encephalomyelitis, glatiramer acetate

\section{Introduction}

Multiple sclerosis (MS) is a disease of the central nervous system (CNS), characterized pathologically by inflammation, demyelination, axonal damage and neuronal loss[1]. These events are triggered by heterogenous myelin-reactive peripheral immune cells infiltrating the blood-brain-barrier (BBB) [2]. The most widely used animal model for MS is experimental autoimmune encephalomyelitis (EAE), a murine model which presents with CNS inflammation, demyelination, axonal transection, and neurological 


\section{Running Title: GA reduces ER \& mitochondrial stress in EAE}

impairment due to similar infiltration of auto-reactive cells [3-7]. The currently available diseasemodifying therapies for MS have not been proven to prevent or reverse long-term neurodegeneration [8,9], although they have been shown to reduce inflammation [10]. Yet, pathological axonal damage [6] and neuronal loss [11] in the brain and spinal cord atrophy on magnetic resonance imaging (MRI) are highly correlated with long-term disability and progression more so than inflammation [12]. Therefore, MS research is currently seeking to better understand the mechanisms underlying neurodegeneration in MS in order to identify neuroprotective therapies which promote myelin repair.

Pathological studies of MS lesions have implicated mitochondrial dysfunction in the neurodegeneration in MS [13]. MRI spectroscopy shows changes in N acetyl aspartate levels consistent with decreased mitochondrial function in acute lesions $[14,15]$. The proximate cause of the dysfunction is relatively unknown. Reactive oxygen species (ROS) produced by mitochondria are induced in the pathogenesis of MS and EAE. Enhancement of ROS production following EAE is one of the causes of demyelination and inflammation [16,17]. As a consequence, mitochondrial ROS promotes inflammation and shifts mitochondrial dynamics towards mitochondrial breakdown [18]. Endoplasmic reticulum (ER) stress is also involved in MS lesions and in EAE animals $[19,20]$ Upstream causes of these mitochondrial defects and ER stress are largely unknown. A possible mechanism involves the release of $\mathrm{Ca} 2+$ ions from the ER within the damaged neurons. This intracellular signaling mechanism ultimately induces cell death via a mitochondrial mediated mechanism [21,22]. Abnormal ER mitochondria Ca2+ transfer is observed following axotomy [23] and spinal cord injury [24]. This process not only promotes ER-mitochondria crosstalk in general by increasing the apposition of the ER and mitochondria at the mitochondriaassociated membrane (MAM) [25-27], but could also promote neuronal death, thus is involved in the MS pathology [28]. Mitochondrial metabolism and ER stress are two of the most metabolically active cellular processes, playing a crucial role in regulating inflammation. Furthermore, mitochondrial dysfunction with reduced nicotinamide dinucleotide $(\mathrm{NAD}+)$ appears to play a role in neurodegeneration in $\mathrm{MS}$ pathogenesis [29-33]. Previously, we studied transgenic EAE mice with neuron specific overexpression 


\section{Running Title: GA reduces ER \& mitochondrial stress in EAE}

of SIRT1, an NAD dependent enzyme, and found evidence supporting a protective role of SIRT1 in EAE [34] prevented or altered the phenotype of inflammation in spinal cords; as a result, demyelination and axonal injury were reduced. SIRT1 also regulates mitochondrial function [35], ER stress [36] and inflammation [37]. This has shifted research focus towards the effects of inflammation on mitochondrial dysfunction, ER stress and NAD associated SIRT1 pathway.

Glatiramer Acetate (GA), commonly known as copaxone, is an approved immunomodulatory diseasemodifying drug for MS treatment [38]. GA mediates remyelination by altering T-cell differentiation and inducing a shift towards Th2/3 cells and neurotrophic factors that can penetrate the blood-brain-barrier (BBB) and accumulate in the CNS [39-42]. To date, the effects of GA on ER stress, mitochondrial dysfunction and NAD+ SIRT1 in the CNS of EAE mice has not been studied, so we set out to investigate this. Using routine histology, immunohistochemistry, western blotting, and electron microscopy, we discovered that GA can regulate ER stress, mitochondrial function and dynamics, and NAD+ dependent pathways by inhibiting neuroinflammation. Our findings elucidate that targeting neuroinflammation associated with ER stress, mitochondrial dysfunction and NAD regulated mechanism through a peripherally acting drug can be neuroprotective in MS.

\section{Methods}

2.1 Animals and Drug Female C57B1/6J mice were obtained from The Jackson Laboratory (Bar Harbor, ME, USA). Mice were housed in our facilities under pathogen-free conditions at the University of Maryland, School of Medicine, Baltimore. Mice were maintained and treated following NIH guidelines and the Institutional Animal Care and Use Committee-approved protocol. EAE was induced in eight week old female C57Bl/6 mice with $0.2 \mathrm{mg}$ of the myelin oligodendrocyte glycoprotein (MOG) 35-55 peptide in complete Freund's adjuvant (CFA) followed by pertussis toxin injections as we have described previously [34]. Mice were scored daily by blinded raters using a standard impairment scale as described 


\section{Running Title: GA reduces ER \& mitochondrial stress in EAE}

previously[34]. GA (TEVA Neuroscience) was administered subcutaneously at a dose of

$125 \mu \mathrm{g} / \mathrm{mouse} /$ day in a $200 \mu \mathrm{l}$ vehicle of phosphate-buffered saline (PBS). The mice were randomized to vehicle-treated EAE (EAE), GA-treated EAE (EAE+GA) and vehicle-treated age matched normal female mice. All mice received a single injection daily starting from the day of disease onset among the EAE mice (Score $\geq 1)$.

2.2 Tissue Pathology: Mice were euthanized and spinal cords and brains were removed and prepared for histology as described previously [34]. Immunohistochemical and immunofluorescence studies were carried out as described previously [34]. The primary antibodies used are listed in Table 1.

Table 1: List of antibodies used in the study.

\begin{tabular}{|c|c|c|c|}
\hline Antibody & Target & Vendor & Concentration \\
\hline Anti-IFN-y & Pro-inflammatory Cytokine & Bioss, Woburn, MA, USA & $1: 100(\mathrm{IHC})^{*}$ \\
\hline Anti-IL-17 & Pro-inflammatory Cytokine & $\begin{array}{l}\text { Santa Cruz Biotechnology, } \\
\text { Santa Cruz, CA, USA }\end{array}$ & $1: 50(\mathrm{IHC})$ \\
\hline Anti-MBP & Myelin Basic Protein & $\begin{array}{l}\text { Abcam, Cambridge, MA, } \\
\text { USA }\end{array}$ & $1: 500(\mathrm{IF})^{* *}$ \\
\hline Anti-MFN-2 & Mitofusin-2 & $\begin{array}{l}\text { Abcam, Cambridge, MA, } \\
\text { USA }\end{array}$ & $1: 400(\mathrm{IF})$ \\
\hline Anti-Fis-1 & Mitochondrial Fusion & BioVision, Milpitas, CA, USA & $1: 100(I F)$ \\
\hline Anti-DNM1-L & Dynamin-like protein & $\begin{array}{l}\text { LifeSpan Biosciences, } \\
\text { Seattle, WA, USA }\end{array}$ & $1: 600(\mathrm{IHC})$ \\
\hline Anti-PGC1- $\alpha$ & $\begin{array}{l}\text { Peroxisome proliferator- activated } \\
\text { receptor gamma coactivator } 1 \text {-alpha }\end{array}$ & $\begin{array}{l}\text { Abcam, Cambridge, MA, } \\
\text { USA }\end{array}$ & $1: 500(\mathrm{IHC})$ \\
\hline
\end{tabular}


bioRxiv preprint doi: https://doi.org/10.1101/2021.05.04.442578; this version posted May 4, 2021. The copyright holder for this preprint (which was not certified by peer review) is the author/funder. This article is a US Government work. It is not subject to copyright under 17 USC 105 and is also made available for use under a CCO license.

\section{Running Title: GA reduces ER \& mitochondrial stress in EAE}

\begin{tabular}{|c|c|c|c|}
\hline Anti-FOXO-1 & Forkhead box protein 1 & $\begin{array}{l}\text { Santa Cruz Biotechnology, } \\
\text { Santa Cruz, CA, USA }\end{array}$ & $1: 100(\mathrm{IHC})$ \\
\hline Anti-TFAM & Mitochondrial transcription factor A & Bioss, Woburn, MA, USA & 1:1000(IHC) \\
\hline $\begin{array}{l}\text { Anti-Cytochrome C } \\
\text { Oxidase }\end{array}$ & Cytochrome $\mathrm{C}$ release & $\begin{array}{l}\text { Abcam, Cambridge, MA, } \\
\text { USA }\end{array}$ & 1:50 (IHC) \\
\hline Anti-SIRT-1 & Sirtuin 1 & $\begin{array}{l}\text { LifeSpan Biosciences, } \\
\text { Seattle, WA, USA }\end{array}$ & 1:500(IHC) \\
\hline Anti-SIRT-3 & Sirtuin 3 & $\begin{array}{l}\text { Santa Cruz Biotechnology, } \\
\text { Santa Cruz, CA, USA }\end{array}$ & $1: 100(\mathrm{IHC})$ \\
\hline Anti-NAMPT & $\begin{array}{l}\text { Nicotinamide } \\
\text { phosphoribosyltransferase }\end{array}$ & $\begin{array}{l}\text { Novus Biologicals, Littleton, } \\
\text { CO, USA }\end{array}$ & $1: 100(\mathrm{IHC})$ \\
\hline Anti- BAX & Pro apoptotic marker & $\begin{array}{l}\text { Life Span Biosciences, } \\
\text { Seattle, WA, USA. }\end{array}$ & $1: 2000(\mathrm{IHC})$ \\
\hline Anti- $\mathrm{CHOP}$ & $\begin{array}{l}\text { Endoplasmic reticulum stress } \\
\text { marker }\end{array}$ & $\begin{array}{l}\text { Life Span Biosciences, } \\
\text { Seattle, WA, USA }\end{array}$ & $1: 500(\mathrm{IHC})$ \\
\hline Anti- PERK & $\begin{array}{l}\text { Endoplasmic reticulum stress } \\
\text { marker }\end{array}$ & $\begin{array}{l}\text { Abcam, Cambridge, MA, } \\
\text { USA }\end{array}$ & $1: 500(\mathrm{IHC})$ \\
\hline Anti- PARKIN & Mitophagy marker & $\begin{array}{l}\text { Novus Biologicals, Littleton, } \\
\text { CO, USA }\end{array}$ & $1: 500(\mathrm{IHC})$ \\
\hline Anti- PINK1 & Mitophagy marker & $\begin{array}{l}\text { Abcam, Cambridge, MA, } \\
\text { USA }\end{array}$ & $1: 200(\mathrm{IHC})$ \\
\hline Anti-VDAC-1 & $\begin{array}{l}\text { Mitochondrial Membrane protein } \\
\text { marker }\end{array}$ & $\begin{array}{l}\text { Abcam, Cambridge, MA, } \\
\text { USA }\end{array}$ & $1: 500(\mathrm{IHC})$ \\
\hline Anti-PACS2 & $\begin{array}{l}\text { Mitochondrial Membrane protein } \\
\text { marker }\end{array}$ & Boster, Pleasanton, CA, USA & $1: 500(\mathrm{IHC})$ \\
\hline
\end{tabular}

\subsection{TUNEL assay for apoptotic cell death:}




\section{Running Title: GA reduces ER \& mitochondrial stress in EAE}

ApopTag Peroxidase Kit (Chemicon International, Temecula, CA) was used to assess the extent of cell death in the spinal cord sections of EAE animals and GA treated animals. Briefly, all the slides were deparaffinized with xylene and rehydrated through graded alcohols to water, washed in $0.01 \mathrm{M}$ PBS then pretreated with a protein-digesting enzyme for $15 \mathrm{~min}$ and then washed with water for $2 \mathrm{~min}$. slides were treated with $3 \%(\mathrm{v} / \mathrm{v})$ hydrogen peroxide for 5 min followed by washing with PBS. Terminal deoxynucleotidyl transferase (TdT) enzyme was added to the pre-equilibrated spinal cord sections and incubated for $1 \mathrm{~h}$ at $37^{\circ} \mathrm{C}$. Stop-buffer was added to the slide and agitated for $15 \mathrm{sec}$ followed by $10 \mathrm{~min}$ incubation at room temperature. After washing three times with PBS for 1 min each, anti-digoxigenin peroxidase conjugate was added to the slides and incubated for $30 \mathrm{~min}$. After slides were washed twice with PBS, freshly prepared peroxidase substrate 3,3'-diaminobenzidine was added to the slides and kept for $6 \mathrm{~min}$ and then slides were washed with water three times. Slides were counterstained with $0.5 \%(\mathrm{w} / \mathrm{v})$ DAB for 5 min followed by washing with water and then $100 \%$ n-butanol. After 10 min, cells were dehydrated in xylene for 2 min and then mounted with glass coverslip. Experiments were conducted in triplicates and the ApopTag-positive cells was determined by counting cells under light microscopy

\subsection{Analysis of histological images using Image $\mathbf{J}$ :}

Image $\mathrm{J}$ was used for histological quantification by a blinded observer. Cell infiltration was quantitated by counting the number of positive quadrants with inflammation, and then expressed as a percentage over the total number of quadrants examined in the histogram as reported previously[34]. Demyelination was quantitated using LFB staining and MBP staining as described previously[34]. The cell labeling experiments (IL-17, IFN- $\gamma$, Cytochrome C, PINK-1, PERKIN, DNM1-L, PGC-1alpha, TFAM, CHOP, PERK, PACS-2, VDAC-1, NAMPT, SIRT-1, SIRT-3, FOXO-1, BAX, MBP, NFM-2, Fis-1 ) were quantified based on the number of positive cells/field (200X or 400X). All fields covering the entire white matter (10-12 fields/section) and gray matter (5-7 fields/section) were analyzed from each spinal cord. The cell counting and data analysis were performed by an examiner blinded to treatment assignment. 
Running Title: GA reduces ER \& mitochondrial stress in EAE

\subsection{Electron Microscopy:}

Spinal cords were excised and fixed with a solution of $2 \%$ paraformaldehyde, $2.5 \%$ glutaraldehyde, in 0.1M PIPES buffer (pH 7) at $4^{\circ} \mathrm{C}$ overnight. Specimens were washed with 0.1M PIPES buffer (pH 7), treated with 50mM glycine in $0.1 \mathrm{M}$ PIPES buffer for 15 minutes and washed again with $0.1 \mathrm{M}$ PIPES buffer. Tissue pieces were then post-fixed in $1 \%$ osmium tetroxide, $1.5 \%$ potassium ferrocyanide in $0.1 \mathrm{M}$ PIPES buffer for $60 \mathrm{~min}$, washed and followed by en bloc staining with 1\% (w/v) uranyl acetate for 60 min. After washing, specimens were dehydrated using a serial graded ethanol solution $(30 \%, 50 \%, 70 \%$, $90 \%$ and $100 \%$ ) and then $100 \%$ acetone. After dehydration, specimens were infiltrated and embedded in Araldite resin (Electron Microscopy Sciences, PA) following manufacturer's recommendation. Ultra-thin sections $\sim 70 \mathrm{~nm}$ thickness were cut on a Leica UC6 ultramicrotome (Leica Microsystems, Inc., Bannockburn, IL) and collected onto copper grids and examined in an FEI Tecnai T12 transmission electron microscope (FEI. Co., Hillsboro, OR) operated at $80 \mathrm{kV}$. Digital images were acquired by using a bottom mount CCD camera (Advanced Microscopy Techniques, Corp, Woburn, MA) and AMT600 software. A minimum of 3 different grids were examined for each animal from each group $(\mathrm{N}=5)$. All the grids were examined at x1100 for identification of white matter and gray matter and then examined at higher magnification for mitochondrial structure and endoplasmic reticulum integrity $(\mathrm{x} 6,500$ and $\mathrm{x}$ $11,000)$.

\subsection{Statistical analysis:}

Data analysis was performed using Prism software (Graph Pad, San Diego, CA) and groups were compared using one-way analysis of variance (ANOVA) with Fisher's protected least significant difference (PLSD) post hoc test at a 95\% confidence interval. All results were presented as mean \pm standard error of mean (SEM) of separate experiments ( $n \geq 5)$. Differences were considered significant at $p \leq 0.05$. 


\section{Running Title: GA reduces ER \& mitochondrial stress in EAE}

2.7 Data availability: The primary data upon which this manuscript is based is available from the corresponding author upon reasonable request. This study was not pre-registered.

\section{Results}

\subsection{GA treatment improves clinical score, reduces demyelination, and suppresses inflammation in EAE mice:}

Clinical Score: All mice developed clinical symptoms at about post immunization day (p.i.d.) $9( \pm 2.0)$ and without treatment average clinical scores remained elevated through p.i.d. 30 (Figure 1A1). GA treatment started at the time of symptom onset reduced the severity of disease within three days and scores remained significantly lower than the untreated EAE group with average clinical scores over days 10 to 30 of $0.57 \pm 0.06$ compared with $1.8 \pm 0.15 ; \mathrm{P}<0.0001$ (Figure $1 \mathrm{~A} 2$ ).

Demyelination was assessed in sections of spinal cord using Luxol Fast Blue (LFB) staining (Figures 1B1-1B3). Quantitative analysis of the extent of demyelination shows $40.99 \pm 2.7 \%$ of white matter with myelin loss (demyelination) in EAE as compared to $8.62 \pm 2.4 \%$ in $\mathrm{EAE}+\mathrm{GA}(\mathrm{P}<0.0001)$ (Figure 1B4). These results were confirmed using immunohistochemical analysis with antibodies against myelin basic protein (MBP) (Figures 1B5-1B7). Quantitative analysis of the percentage of white matter with MBP loss showed significant loss in EAE that was reduced by GA treatment (Figure 1B8). The changes in demyelination were confirmed qualitatively by transmission electron microscopy at 6500x (Figure 1B91B11).

Production of pro-inflammatory cytokines was examined by immunohistochemical staining for interleukin-17 (IL-17) and interferon-gamma (IFN-g) (Figure 1C). The number of cells expressing IL-17 was increased in EAE $\mathrm{P}>0.05$ but reduced by GA treatment, $\mathrm{P}<0.05$ (Figure 1C4). The number of cells expressing IFN-g was increased in $\mathrm{EAE} \mathrm{P}<0.001$ but reduced by GA treatment $\mathrm{P}<0.001$ (Figure 1C8). 


\section{Running Title: GA reduces ER \& mitochondrial stress in EAE}

$\underline{\text { Inflammatory cell infiltration was examined in H\&E stained sections of spinal cord from mice }}$ euthanized on day 30 (Figure 1C9-C11). While normal mice did not have cellular infiltration, EAE mice had $47.09 \pm 1.86 \%$ of white matter with inflammation compared to $12.95 \pm 2.34 \%$ in EAE+GA (Figure $1 \mathrm{C} 12, \mathrm{P}<0.0001)$

Fig 1: Glatiramer Acetate treatment improves clinical score, reduces demyelination, and suppresses inflammation in EAE mice. (A) The clinical severity of EAE disease was reduced by GA treatment. (1A) Graph of mean EAE scores for both groups of mice over a 30-day period. (1B) Mean score for all animals in each group during the disease phase (days 10-30). The mean score was significantly lower in the GA treated EAE $(\mathrm{EAE}+\mathrm{GA})$ mice $(\mathrm{P}<0.0001)$ compared to untreated EAE mice. $\mathrm{N}=20$ /group, t-test. (B) Demyelination in EAE was reduced by GA treatment. Demyelination was assessed on LFB-stained (B1 - B3) and MBP stained (B5 - B7) sections of spinal cord white matter. Original magnification was $\mathrm{x} 400$. B4 and $\mathrm{B} 8$ are graphs of the number of quadrants with demyelination expressed as a percentage of the total number of quadrants examined $(n=4)$, with statistics based on the t-test. B9 - B11 show transmission electron microscographs (TEM) at 6500x magnification in normal mouse (B9) untreated EAE mice (B10) and EAE with GA treatment (B11). These images are representative of at least 3 grids from each of five mice in each group. (C) Inflammation in EAE was reduced by GA treatment. Inflammation was assessed in spinal cord white matter by $(\mathrm{C} 1-\mathrm{C} 4)$ cells staining positively for antibodies to IL-17 and (C5 - C8) cells staining positively for IFN $\gamma$. Original magnification was x400. Hematoxylin and eosin (H\&E) stained sections show infiltration of mononuclear cells in white matter of $\mathrm{EAE}(\mathrm{C} 10)$ and $\mathrm{EAE}+\mathrm{GA}(\mathrm{C} 11)$ mice. However, the number of inflammatory pockets and inflammatory cells is fewer in EAE+GA compared to EAE. Normal (C9) mice show no inflammatory infiltrates. (C12). The number of positive quadrants with inflammation was scored and expressed as a percentage of the total number of quadrants $(H \& E ; n=4)$. Bar graphs show cell counts and statistical comparisons based on one-way ANOVA.

\subsection{GA reduces ER Stress in EAE spinal cord:}




\section{Running Title: GA reduces ER \& mitochondrial stress in EAE}

We examined ER stress by assessing cells expressing CCAAT-enhancer-binding protein homologous protein (CHOP), which is induced by ER stress and is a mediator of apoptosis [43]. The number of cells positive for CHOP was increased in EAE but decreased in EAE mice treated with GA (Figure 2A1-2A3). Figure 2A4 shows the quantitative analysis of $\mathrm{CHOP}$ expression, which was significantly decreased in EAE + GA compared to EAE $(\mathrm{P}<0.01)$. Next we examined ER stress in sections of the spinal cord using antibodies to protein kinase RNA-like endoplasmic reticulum (PERK) a component of mitochondriaassociated ER membranes[44]. The number of cells positive for the PERK was increased in EAE but decreased in EAE mice treated with GA (Figure 2B1 - 2B3). Figure 2B4 shows the quantitative analysis of GFAP expression, which was significantly decreased in EAE+GA compared to EAE. Finally, we examined the structural integrity of the endoplasmic reticulum using transmission electron microscopy with 6500x magnification and found ER disruption in EAE that was not present in normal or GA treated EAE (Figure 2C1-2C3).

Fig 2: GA reduces ER stress in EAE spinal cord. Endoplasmic reticulum stress was assessed with antibodies to CHOP (A1 - A4) and PERK (B1 - B4). Panels 1 - 3 show representative staining patterns in each treatment group for each antibody. Original magnification: X400. Panel 4 in each case shows a graph of the mean number of positive cells per field for each treatment group $(\mathrm{CHOP}: \mathrm{n}=4$; PERK: $\mathrm{n}=$ 4) with intergroup comparisons based on a one-way ANOVA. Transmission electron microscopic examination $(\mathrm{C} 1-\mathrm{C} 3)$ showed vesiculated endoplasmic reticulum, irregularly arranged and disrupted endoplasmic reticulum (arrows) in untreated EAE (C2) while regular and parallel organized endoplasmic reticulum in normal (C1) and GA treated EAE mice (C3).

\subsection{GA treatment improves mitochondrial function, fission/fusion, and biogenesis in EAE spinal} cord:

Mitochondrial integrity was assessed by immunohistochemical and immunofluorescent staining. Function was assessed using expressions of PINK-1 and PARKIN. PINK-1 accumulates on poorly functioning 


\section{Running Title: GA reduces ER \& mitochondrial stress in EAE}

(depolarized) mitochondria allowing PARKIN to bind, targeting the mitochondria for autophagy [45].

PINK-1 and PARKIN were increased in untreated EAE but not in EAE treated with GA (Figure 3A1-3A3 and 3B1-3B3), confirming lost mitochondrial integrity in EAE and revealing that GA can restore this. Figures 3A4 and 3B4 shows the quantitative analysis of PGC1- $\alpha$ expression

Mitochondrial biogenesis was examined in spinal cords for cells expressing PGC1- $\alpha$, a mediator of mitochondrial biogenesis[46], by immunohistochemistry (Figure 3C1 - 3C3). Figure 3C4 shows the quantitative analysis of PGC1- $\alpha$ expression, which was significantly reduced in EAE mice $\mathrm{P}<0.01$, but restored back to normal levels in EAE mice treated with GA $(\mathrm{P}<0.01)$.

Morphological changes in mitochondria were assessed directly by transmission electron microscopy at 6500X magnification (Figure D1-D3). Mitochondrial fission, cristae damage and loss, mitochondrial membrane damage and disappearance, and changes in size and shape were seen in EAE mice but not in the tissues from normal mice or EAE mice treated with GA. Mitochondrial fusion was not seen.

Mitochondrial fusion, which is reduced under conditions of ER stress, was assessed by immunohistofluorescence staining (Figure 3E1-3E3) and western blot analysis for MFN-2, a marker of fusion[47]. The number of cells positive for the MFN-2 was reduced in $\mathrm{EAE}(\mathrm{P}<0.05)$ but not in $\mathrm{EAE}$ mice treated with GA compared to normal mice $(\mathrm{P}<0.05)$ (Figure 3E4).

Mitochondrial fission, which is increased under conditions of mitochondrial stress, was assessed by immunofluorescence staining for FIS-1[48] and both immunohistochemistry and western blot analysis for DNM1-L (Figure 3F1-3F3 and 3G1-3G3, 3G5)[49]. The number of cells expressing FIS-1 did not change in the EAE mice but significantly decreased in EAE mice treated with GA compared to control EAE mice $(\mathrm{P}<0.05)$ (Figure 3F4). The number of cells expressing DNM1-L significantly increased in the EAE mice $(\mathrm{P}<0.01)$ but decreased to normal levels in $\mathrm{EAE}+\mathrm{GA}(\mathrm{P}<0.01)$ (Figure 3G4).

\section{Fig 3: GA treatment improves mitochondrial function, fission/fusion, and biogenesis in EAE mice.}

$(\mathrm{A}, \mathrm{B})$ EAE caused loss of mitochondrial integrity that was reduced by GA treatment. Mitochondrial 


\section{Running Title: GA reduces ER \& mitochondrial stress in EAE}

integrity was assessed by staining for PINK-1 (A1 - A4) and staining for PARKIN (B1 - B4). Panels 1 - 3 show representative staining patterns in each treatment group for each antibody. Original magnification: X400. Panel 4 in each case shows a graph of the mean number of positive cells per field for each treatment group $(n=4)$ with intergroup comparisons based on a one-way ANOVA. $(C, D)$ Changes in mitochondrial biogenesis and morphology in EAE are reduced by GA treatment. Mitochondrial biogenesis was assessed with antibody to PGC1- $\alpha$, a regulator of mitochondrial biogenesis $(\mathrm{C} 1-\mathrm{C} 4)$ Panels $1-3$ show representative staining patterns in each treatment group for each antibody. Original magnification: X400. Panel 4 shows a graph of the mean number of positive cells per field for each treatment group $(n=3 ; n=4)$ with intergroup comparisons based on a one-way ANOVA. Mitochondrial morphology was assessed by transmission electron microscopy (TEM) at 6500x magnification. Morphological changes were observed in mitochondria in EAE (D2) that were reduced by treatment (D3) compared to the mitochondria in normal mice (D1). In EAE many of the mitochondria showed mitochondrial membrane disruption with disruption or loss of cristae and changes in mitochondrial size and shape with increased fission. The results shown are typical of at least 3 grids for each mouse $(n=5)$. (E-G) EAE caused changes in mitochondrial dynamics that were reduced by GA treatment. Mitochondrial fusion was assessed by staining with antibody to Mitofusin - 2 (MFN-2) (E1 E4) and mitochondrial fission was assessed with antibody to FIS-1 (F1 - F4) and DNM1-L (G1 - G4). Panels 1 to 3 show representative staining patters in each treatment group with each antibody. Original magnification was $x 400$. Panel 4 in each case shows a graph of the mean number of positive cells per field for each treatment group (MFN-2, $n=4$; FIS-1, $n=3$; DNM1-L, $n=3$ ) with intergroup comparisons based on a one-way ANOVA. (E5) Western Blot analysis of mitochondrial fraction of spinal cord shows an increase in MFN-2 in the EAE+GA mice compared to EAE and Normal. (G5) Western Blot analysis of mitochondrial fraction of spinal cord shows a decrease in DNM1-L in the EAE+GA mice compared to EAE and Normal.

\subsection{GA regulates changes in the mitochondria associated membrane (MAM) and increases}


Running Title: GA reduces ER \& mitochondrial stress in EAE

activity of the NAD+ dependent pathway in EAE spinal cord:

Mitochondria and the endoplasmic reticulum interact through a specialized domain on the ER called the mitochondria-associated membranes (MAM). Several proteins are located in MAMs, including PACS-2, VDAC-1[50,51]. We examined PACS-2 and VDAC-1 expressions in EAE using immunohistochemistry and evaluated the effect of GA treatment (Figure 4A1-4A3 and 4B1-4B3). Numbers of cells expressing PACS-2 and VDAC-1 (Figure 4A4 and 4B4) were increased in EAE $(\mathrm{P}<0.05)$ but returned toward normal in GA treated mice $(\mathrm{P}<0.05)$.

Fig 4: GA regulates changes in mitochondria associated membrane (MAM) associated with EAE in the spinal cord. Antibodies to PACS2 (A1-A4) and VDAC-1 (B1-B4) were studied as markers of the MAM. (A1 - A3 and B1 - B3) show the antibody staining in each treatment group with each antibody at an original magnification of $\mathrm{x} 400$. (A4 and B4) show quantitation of staining in each treatment group with comparisons by one-way ANOVA $(n=5)$.

Because of the importance of NAD regulation in mitochondrial function and cell survival[52] we examined expression of Nicotinamide phosphoribosyltransferase (NAMPT), the rate limiting enzyme in NAD biosynthesis[53], and Sirtuins 1 and 3 which are NAD dependent protein deacetylases[54] which play a role in mitochondrial dynamics. Staining for NAMPT, SIRT-1 and SIRT-3 and was reduced in EAE compared to normal (Figures 5A2, 5B2, 5C2). Treatment with GA partially restored levels of NAMPT, SIRT-1 and SIRT-3 (Figures 5A3, 5B3, 5C3). Figures 5A4, 5B4 and 5C4 show the quantitative analysis of NAMPT, SIRT-1 and SIRT-3 expressions.

Fig 5: GA increased the activity of NAD-dependent pathways. NAD + dependent pathways were assessed with antibodies to NAMPT (A1 - A4), Sirt-1 (B1 - B4), and Sirt-3 (C1 - C4). For each antibody, panels $1-3$ show typical staining in each treatment group at an original magnification of $\mathrm{x} 400$ and panel 4 is a graph of the quantification of positive cells in the spinal cord with comparisons made using one-way ANOVA $(n=4)$. 


\section{Running Title: GA reduces ER \& mitochondrial stress in EAE}

\subsection{GA reduces apoptosis in EAE spinal cord:}

Because of mitochondrial involvement in programmed cell death we assessed apoptosis in EAE using the TUNEL assay. TUNEL cells were significantly increased in EAE $(\mathrm{P}<0.001)$ but lower in $\mathrm{EAE}+\mathrm{GA}$ spinal cords $(\mathrm{P}<0.001)$ (Figure 6A1-6A3). Figure 6A4 shows the quantitative analysis of TUNEL positive cells in the gray matter of these spinal cords. To evaluate activation of the apoptosis pathway we examined the expression of FOXO-1, a transcription factor regulated by AKT and implicated in apoptosis [55] in part by the regulation of Bax, an activator of apoptosis[56]. FOXO-1 expressing cells were increased in EAE $(\mathrm{P}<0.05)$ but normal in GA treated mice $(\mathrm{P}<0.001)$ (Figure 6B1-B4). BCL-2 expressing cells were increased in EAE $(\mathrm{P}<0.05)$ but lower after GA treatment $(\mathrm{P}<0.05)$ (Figure 6C1-4). Staining for cytochrome-C expression in spinal cord sections was used as a measure of apoptosis triggered by decreased mitochondrial integrity[57](Figure 6A1-6A3). Cytochrome C expressing cells were increased in $\mathrm{EAE}(\mathrm{P}<0.001)$ but was reduced back to normal levels in GA treated mice $(\mathrm{p}<0.001)$ (Figure 6A4).

Fig 6: GA reduces apoptosis in EAE spinal cord. Apoptosis was assessed in spinal cord tissue with in situ TUNEL staining (A1 - A4) and with antibodies to FOXO-1 (B1 - B4), BAX (C1 - C4), and Cytochrome C (D1-D4). Panels 1 - 3 show typical staining patterns for each treatment group at an original magnification of $\mathrm{x} 400$ while panels 4 provide quantitation of numbers of positive cells in each group $(n=4)$ with comparisons using one-way ANOVA.

\section{Discussion}

In this study, we investigated for the first time the effects of GA, a polypeptide-based approved drug for the treatment of MS, on ER stress and mitochondrial dysfunction and NAD related pathways, along with neuroinflammation and demyelination, in the spinal cord of EAE mice. Our principle findings are: (i) as expected, GA improved clinical score, reduced inflammatory activity, and promoted remyelination; (ii) 


\section{Running Title: GA reduces ER \& mitochondrial stress in EAE}

GA reduced ER stress; (iii) GA improves mitochondrial function, reduces mitochondrial fission, increases mitochondrial fission, and increases mitochondrial biogenesis; (iv) GA regulated changes in mitochondrial associated membranes; (v) GA increases activity of the NAD+ dependent pathway; and (vi) GA reduces apoptosis.

Research thus far has demonstrated that GA exerts its immunomodulatory effects by altering T-cell differentiation through promotion of Th2-polarized GA-reactive CD4+ T-cells [58]. Furthermore, induction of Th2 cells in the periphery during GA treatment leads to reduced inflammation, and in turn promotes remyelination and neuronal survival [59]. Figure 1 of our research confirms these findings. Specifically, we showed that GA downregulated expression of IFN-y, which promotes myelin damage by stimulating inflammation. It is important to note that GA is degraded in the periphery and cannot cross the blood brain barrier, so the spinal cord findings that we present demonstrate GA's in situ bystander effect [42]. Furthermore, GA treatment increased Th2 cytokine IL-10 expression. It is recently reported that GA treatment causes a switch from pro-inflammatory microglia and astrocytes to anti-inflammatory phenotypes of those CNS cells that are involved in MS pathogenesis [60].

ER stress is involved in various intracellular physiological functions such as protein folding, calcium homeostasis, lipid metabolism, cell differentiation, and protein translocation [61]. ER stress is characterized by the accumulation of misfolded proteins, resulting in chronic perturbations to ER homeostasis [62]. Increasing evidence suggests that ER stress, which is caused by the accumulation of unfolded or misfolded proteins in the ER, plays a role in inflammatory diseases, including MS and EAE $[63]$.

The unfolded protein response (UPR) is an evolutionary conserved process that is activated in order to restore ER homeostasis by correcting protein-folding machinery. The UPR has three main arms led by ER-transmembrane proteins: PERK, IRE1, and ATF6. In this study, we focused specifically on PERK and its downstream apoptotic gene CHOP. Although ER stress initially acts as self-preservation, chronic 


\section{Running Title: GA reduces ER \& mitochondrial stress in EAE}

ER stress and activation of the UPR leads to cellular apoptosis [64]. It is widely known that the UPR is activated in both MS and EAE lesions, induced by elevated levels of proinflammatory mediators, and contributes to disease progression [65]. Specifically, previous studies have demonstrated that EAE, including spinal cord tissue, exhibits upregulated levels of p-PERK and CHOP in oligodendrocytes, $\mathrm{T}$ cells, astrocytes, and macrophages/ microglia [50,65-67]. We confirmed the increases in PERK and CHOP using immunostaining and showed that they were reversed by immunomodulating treatment with GA. In our study, we found that GA reversed the activation of PERK and CHOP in the EAE model, and this was supported by our electron microscopy that presented restored ER structure similar to that of wild type mice. We suggest that this is due to GA's ability to mediate neuroinflammation, specifically IFN-y. IFN-y has previously been shown to induce PERK activation and its downstream translation initiation factor 2 (eIF2 $\alpha$ ), and IFN-y induced apoptosis in rat oligodendrocytes is associated with ER stress [68]. Targeting the PERK-eIF2 $\alpha$ pathway has been reported as an ideal strategy for protecting oligodendrocyte protection in MS, and we show for the first time that GA may be able to [69]. Other chemical compounds that have been shown to activate this pathway and are neuroprotective in EAE and MS include salburnal [70] and guanabenz [71]. Future studies with GA should investigate its cytoprotective effects on other branches of the UPR in specifically oligodendrocytes and neurons. It is demonstrated that ER stress generated in murine astrocytes encourages PERK-dependent inflammatory signaling in vitro, suggesting that astrocytes themselves are potential contributors to neurotoxic inflammation in the face of ER dysfunction $[66,72]$. Particularly we provided evidence of the link between the effector cytokine molecules in regulating ER stress by GA treatment in EAE mice. Furthermore, these studies suggest that controlling balance of ER stress and inflammatory response can serve as an important therapeutic mechanism of GA for regulating EAE and perhaps MS.

Mitochondrial dysfunction is a pathological hallmark in EAE and MS lesions, and it is well associated with ER stress [19,20]. Therefore, we were interested in examining changes in mitochondrial dynamics to determine if GA would reverse such changes, possibly through attenuation of the ER-stress induced UPR. 


\section{Running Title: GA reduces ER \& mitochondrial stress in EAE}

The PERK-ATF4-CHOP pathway exerts regulatory effects on the expression of Parkin, a critical regulator in mitochondrial dynamics (Sarrabeth Stone 2015). Parkin plays a role in mitochondrial dynamics [73,74], bioenergetics [75,76], and mitophagy [45]. Parkin also is known to modulate MAMs, which we assessed by staining for PACS2 and VDAC1, to maintain calcium transfer between the ER and mitochondria [75]. Haile et al recently showed that during MS progression, ER stress is strongly associated with the upregulation of Rab32, a GTPase that regulates MAMs, and contributes to neuronal death. We found that both the PINK1/Parkin pathway and MAMs was upregulated in the EAE model as found previously [77], and GA reversed this, likely by suppressing the PERK branch of the UPR.

The UPR, PINK1/Parkin pathway, and MAMs all play a role in mitochondrial fission/fusion processes. ER-stress induced PERK regulates the Drp1-Fis1 complex through control of the adaptor protein AKAP121. PINK1 and Parkin promote mitochondrial fission via a Drp1 mediated mechanism [78]. Parkin also negatively regulates mitochondrial fusion via MFN2 by ubiquitination [79] and interestingly, MFN2 deficiency is actually associated with contributing to the UPR response as well as cellular apoptosis because MFN2 plays a role in suppressing PERK [80,81]. Previous studies have also demonstrated that ER-mitochondrial tethering can contribute to the upregulation of Drp-1 mediated fission via formation of constriction sites $[82,83]$. Recently, it was found that Drp1 is activated in experimental models for multiple sclerosis, and inhibition of its pathological hyperactivation is neuroprotective [84]. For the first time, we show that GA reduces mitochondrial fission activity and increases fusion activity in EAE mice, again strongly suggesting that GA targets ER stress and downstream mitochondrial mediators.

To further explore the effects of EAE and treatment of EAE with immunomodulating therapy on metabolism we examined expression of nicotinamide phosphoribosyltransferase (NAMPT), the rate limiting step in the NAD+ salvage pathway which has neuroprotective effects [53] and Sirt 1 and 3, members of the Sirtuin family which are NAD dependent protein deacetylases which are key metabolic sensors in the stress response [54]. Increasing SIRT1 activity, either by treatment with the Sirt activator, 


\section{Running Title: GA reduces ER \& mitochondrial stress in EAE}

resveratrol [85] or by genetic overexpression [34], reduces the clinical and pathological severity of EAE. SIRT3 activates PGC-1 $\alpha$ which stimulates mitochondrial biogenesis and is associated with ROS suppression and neuroprotection[86]. Sirt1 is of further interest because levels are reduced in peripheral blood mononuclear cells during relapses of MS[87] and were restored by treatment with GA[88]. Sirt3 changes are also implicated in MS in that levels were reduced in non-lesioned grey matter from MS brains[89]. We found that expression of NAMPT and Sirt 1 and 3 were reduced in EAE and restored or partially restored to normal levels by treatment with immunomodulating therapy with glatiramer acetate. This suggests that such therapy can restore cells to a more normal metabolic state.

To determine whether the reductions in ER stress, mitochondrial dysfunction and metabolic abnormalities induced by immunomodulatory therapy were associated with reductions in cellular death we examined apoptosis and changes in related pathways. The increased fission processes that we found in the EAE model can contribute to cellular apoptosis by the opening of BAX lined pores and release of cytochrome C [90], and our findings supported this. Using TUNEL staining we found that immunomodulating therapy with GA reduced the high levels of apoptosis is found in untreated EAE. We studied the expression of FOXO transcription factors implicated in the regulation of apoptosis [55] and found increases in EAE which were reversed by GA treatment. We examined the expression of Bax, a BCL-2 family member that promotes apoptosis by contributing to mitochondrial membrane pore formation[56] and found increased levels in EAE which were reversed by GA treatment. These results show that while EAE is associated with an increase in cell death, treatment with the immunomodulating therapy, GA, is associated with a reduction. Our results strengthen the idea that the mitochondrial and ER changes in EAE are part of a coordinated response to metabolic stress caused by inflammation and that immunomodulating therapy can reduce that stress.

We found mitochondrial dysfunction, endoplasmic reticulum stress and disrupted NAD metabolism in the spinal cords of EAE mice. For the first time, we show that GA can potentially reverse these pathological changes, and Figure 7 shows our proposed mechanism. Given that the direct effects of GA 


\section{Running Title: GA reduces ER \& mitochondrial stress in EAE}

are thought to be in the peripheral immune system, it seems likely that the observed changes are an indirect immunomodulatory effect resulting in reduced inflammation in the spinal cord, and thus neuroprotective. The reduction in neuronal apoptosis in EAE upon GA treatment could be a result of ameliorated ER stress, improved mitochondrial function, and regulated NAD metabolism.

Fig 7: Proposed neuroprotective mechanism underlying GA treatment in EAE. During EAE, astroglia and microglia are activated by the infiltrated cells, which includes Th1 cells (T-cells, activated macrophages, B-cells, and Neutrophils) and contribute to a neuroinflammatory environment that damages neurons. As a result, this causes mitochondrial dysfunction and ER stress in neuronal cells. GA induces a shift towards the Th2 response in the periphery, and these Th2 cells cross the blood brain barrier into the CNS. This reduces the inflammatory environment and controls the synergistic ER stress response and mitochondrial dysfunction. By doing so, apoptotic activity in the CNS is downregulated, likely in the myelin producing oligodendrocytes. In turn, disease progression is slowed. CNS AG: CNS antigen; MHC: major histocompatibility complex; TCR: T cell receptor.

\section{Acknowledgements:}

This work was supported by grants from Teva Neuroscience, Inc. and the Department of Veteran's Affairs

\section{Author contributions:}

$\mathrm{C}$ Bever and T Makar designed the study. T Makar induced the EAE in mice, and injected the mice with glatiramer acetate. T Makar and V Nimmagadda euthanized the mice and collected the tissue. V Nimmagadda, P Guda, and S Andhavarapu performed the immunohistochemical staining. P Guda did the western blot analysis. S Ray prepared tissue for electron microscopy, took the pictures, and provided the analysis. T Makar, S Andhavarapu, V Nimmagadda, V Gerzanich, and C Bever analyzed and interpreted the data. S Andhavarapu, T Makar, V Gerzanich, and C Bever drafted the manuscript. All 


\section{Running Title: GA reduces ER \& mitochondrial stress in EAE}

authors critically reviewed and approved the final version of the manuscript.

\section{Competing interests:}

Partial funding for the study was provided by an investigator-initiated grant to Dr. Makar from Teva

Neuroscience, Inc. which markets glatiramer acetate as a treatment for multiple sclerosis. Guda, Ray, Andhavarapu, Nimmagadda, Gerzanich, Simard, and Bever declare no competing interests.

\section{Abbreviations:}

$\mathrm{MS}=$ multiple sclerosis

$\mathrm{CNS}=$ central nervous system

$\mathrm{EAE}=$ experimental autoimmune encephalomyelitis

$\mathrm{UPR}=$ unfolded protein response

$\mathrm{MRI}=$ magnetic resonance imaging

ROS $=$ reactive oxygen species

$\mathrm{ER}=$ endoplasmic reticulum

MAM $=$ mitochondria associated membrane

$\mathrm{NAD}=$ nicotinamide dinucleotide

$\mathrm{GA}=$ glatiramer acetate

$\mathrm{BBB}=$ blood brain barrier

$\mathrm{Ca} 2+=$ calcium ions

$\mathrm{MOG}=$ myelin oligodendrocyte glycoprotein

CFA $=$ complete Freund's adjuvant

PBS $=$ phosphate-buffered saline

IL-17 = interleukin 17

$\mathrm{MBP}=$ myelin basic protein

MFN-2 = mitofusin-2

Fis-1 = fission-1

DNM1-L = dynamin-like protein

PGC1-a = Peroxisome proliferator- activated receptor gamma coactivator 1-alpha

FOXO-1 $=$ Forkhead box protein 1 


\section{Running Title: GA reduces ER \& mitochondrial stress in EAE}

TFAM $=$ mitochondrial transcription factor A

$\mathrm{SIRT}=$ sirtuin

NAMPT $=$ Nicotinamide phosphoribosyltransferase

$\mathrm{BAX}=\mathrm{Bcl}-2$-associated $\mathrm{X}$ protein

$\mathrm{CHOP}=\mathrm{CCAAT}$-enhancer-binding protein homologous protein

PERK $=$ protein kinase RNA-like endoplasmic reticulum

PINK1 $=$ PTEN-induced kinase 1

VDAC-1 = Voltage-dependent anion channel 1

PACS2 $=$ Phosphofurin Acidic Cluster Sorting Protein 2

eIF2 $\alpha=$ eukaryotic translation initiation factor 2 subunit alpha

\section{References:}

1. Lassmann H, Brück W, Lucchinetti CF. The immunopathology of multiple sclerosis: an overview. Brain Pathol. 2007;17: 210-218.

2. Rottlaender A, Kuerten S. Stepchild or Prodigy? Neuroprotection in Multiple Sclerosis (MS) Research. Int J Mol Sci. 2015;16: 14850-14865.

3. Jäger A, Dardalhon V, Sobel RA, Bettelli E, Kuchroo VK. Th1, Th17, and Th9 effector cells induce experimental autoimmune encephalomyelitis with different pathological phenotypes. J Immunol. 2009;183: 7169-7177.

4. Hendriks JJA, Teunissen CE, de Vries HE, Dijkstra CD. Macrophages and neurodegeneration. Brain Research Reviews. 2005. pp. 185-195. doi:10.1016/j.brainresrev.2004.12.008

5. Matthews PM, De Stefano N, Narayanan S, Francis GS, Wolinsky JS, Antel JP, et al. Putting magnetic resonance spectroscopy studies in context: axonal damage and disability in multiple sclerosis. Semin Neurol. 1998;18: 327-336.

6. Trapp BD, Peterson J, Ransohoff RM, Rudick R, Mörk S, Bö L. Axonal transection in the lesions of multiple sclerosis. N Engl J Med. 1998;338: 278-285.

7. Wujek JR, Bjartmar C, Richer E, Ransohoff RM, Yu M, Tuohy VK, et al. Axon Loss in the Spinal Cord Determines Permanent Neurological Disability in an Animal Model of Multiple Sclerosis. Journal of Neuropathology \& Experimental Neurology. 2002. pp. 23-32. doi:10.1093/jnen/61.1.23

8. Lassmann H, van Horssen J, Mahad D. Progressive multiple sclerosis: pathology and pathogenesis. Nat Rev Neurol. 2012;8: 647-656.

9. Soulika AM, Lee E, McCauley E, Miers L, Bannerman P, Pleasure D. Initiation and progression of axonopathy in experimental autoimmune encephalomyelitis. J Neurosci. 2009;29: 14965-14979.

10. Goodin DS. Disease-modifying therapy in multiple sclerosis: update and clinical implications. Neurology. 2008;71: S8-13.

11. Dutta R, Trapp BD. Mechanisms of neuronal dysfunction and degeneration in multiple sclerosis. 


\section{Running Title: GA reduces ER \& mitochondrial stress in EAE}

Prog Neurobiol. 2011;93: 1-12.

12. Jacobsen C, Hagemeier J, Myhr K-M, Nyland H, Lode K, Bergsland N, et al. Brain atrophy and disability progression in multiple sclerosis patients: a 10-year follow-up study. J Neurol Neurosurg Psychiatry. 2014;85: 1109-1115.

13. Witte ME, Mahad DJ, Lassmann H, van Horssen J. Mitochondrial dysfunction contributes to neurodegeneration in multiple sclerosis. Trends Mol Med. 2014;20: 179-187.

14. van Horssen J, Witte ME, Ciccarelli O. The role of mitochondria in axonal degeneration and tissue repair in MS. Mult Scler. 2012;18: 1058-1067.

15. Ciccarelli O, Altmann DR, McLean MA, Wheeler-Kingshott CA, Wimpey K, Miller DH, et al. Spinal cord repair in MS: does mitochondrial metabolism play a role? Neurology. 2010;74: 721-727.

16. Hartung HP, Schäfer B, Heininger K, Toyka KV. Suppression of experimental autoimmune neuritis by the oxygen radical scavengers superoxide dismutase and catalase. Ann Neurol. 1988;23: 453460.

17. Glabiński A, Tawsek NS, Bartosz G. Increased generation of superoxide radicals in the blood of MS patients. Acta Neurol Scand. 1993;88: 174-177.

18. Wang X, Jiang W, Yan Y, Gong T, Han J, Tian Z, et al. RNA viruses promote activation of the NLRP3 inflammasome through a RIP1-RIP3-DRP1 signaling pathway. Nat Immunol. 2014;15: $1126-1133$.

19. Cunnea P, Mháille AN, McQuaid S, Farrell M, McMahon J, FitzGerald U. Expression profiles of endoplasmic reticulum stress-related molecules in demyelinating lesions and multiple sclerosis. Mult Scler. 2011;17: 808-818.

20. Roussel BD, Kruppa AJ, Miranda E, Crowther DC, Lomas DA, Marciniak SJ. Endoplasmic reticulum dysfunction in neurological disease. Lancet Neurol. 2013;12: 105-118.

21. Decuypere J-P, Monaco G, Bultynck G, Missiaen L, De Smedt H, Parys JB. The IP(3) receptormitochondria connection in apoptosis and autophagy. Biochim Biophys Acta. 2011;1813: 10031013.

22. Patterson RL, Boehning D, Snyder SH. Inositol 1,4,5-trisphosphate receptors as signal integrators. Annu Rev Biochem. 2004;73: 437-465.

23. Villegas R, Martinez NW, Lillo J, Pihan P, Hernandez D, Twiss JL, et al. Calcium release from intra-axonal endoplasmic reticulum leads to axon degeneration through mitochondrial dysfunction. $\mathrm{J}$ Neurosci. 2014;34: 7179-7189.

24. Stirling DP, Cummins K, Wayne Chen SR, Stys P. Axoplasmic reticulum $\mathrm{Ca}(2+)$ release causes secondary degeneration of spinal axons. Ann Neurol. 2014;75: 220-229.

25. Bravo R, Vicencio JM, Parra V, Troncoso R, Munoz JP, Bui M, et al. Increased ER-mitochondrial coupling promotes mitochondrial respiration and bioenergetics during early phases of ER stress. $\mathrm{J}$ Cell Sci. 2011;124: 2143-2152.

26. Csordás G, Renken C, Várnai P, Walter L, Weaver D, Buttle KF, et al. Structural and functional features and significance of the physical linkage between ER and mitochondria. J Cell Biol. 


\section{Running Title: GA reduces ER \& mitochondrial stress in EAE}

2006;174: 915-921.

27. Csordás G, Várnai P, Golenár T, Roy S, Purkins G, Schneider TG, et al. Imaging interorganelle contacts and local calcium dynamics at the ER-mitochondrial interface. Mol Cell. 2010;39: 121-132.

28. Simmen T, Lynes EM, Gesson K, Thomas G. Oxidative protein folding in the endoplasmic reticulum: tight links to the mitochondria-associated membrane (MAM). Biochim Biophys Acta. 2010;1798: 1465-1473.

29. Campbell GR, Ohno N, Turnbull DM, Mahad DJ. Mitochondrial changes within axons in multiple sclerosis: an update. Curr Opin Neurol. 2012;25: 221-230.

30. Campbell GR, Worrall JT, Mahad DJ. The central role of mitochondria in axonal degeneration in multiple sclerosis. Mult Scler. 2014;20: 1806-1813.

31. Carvalho KS. Mitochondrial Dysfunction in Demyelinating Diseases. Seminars in Pediatric Neurology. 2013. pp. 194-201. doi:10.1016/j.spen.2013.09.001

32. Kalman B, Laitinen K, Komoly S. The involvement of mitochondria in the pathogenesis of multiple sclerosis. J Neuroimmunol. 2007;188: 1-12.

33. Penberthy WT, Tsunoda I. The importance of NAD in multiple sclerosis. Curr Pharm Des. 2009;15: 64-99.

34. Nimmagadda VK, Bever CT, Vattikunta NR, Talat S, Ahmad V, Nagalla NK, et al. Overexpression of SIRT1 protein in neurons protects against experimental autoimmune encephalomyelitis through activation of multiple SIRT1 targets. J Immunol. 2013;190: 4595-4607.

35. Chandrasekaran K, Anjaneyulu M, Choi J, Kumar P, Salimian M, Ho C-Y, et al. Role of mitochondria in diabetic peripheral neuropathy: Influencing the NAD -dependent SIRT1-PGC-1 $\alpha-$ TFAM pathway. International Review of Neurobiology. 2019. pp. 177-209. doi:10.1016/bs.irn.2019.04.002

36. Guo R, Liu W, Liu B, Zhang B, Li W, Xu Y. SIRT1 suppresses cardiomyocyte apoptosis in diabetic cardiomyopathy: An insight into endoplasmic reticulum stress response mechanism. Int J Cardiol. 2015;191: 36-45.

37. Xie J, Zhang X, Zhang L. Negative regulation of inflammation by SIRT1. Pharmacological Research. 2013. pp. 60-67. doi:10.1016/j.phrs.2012.10.010

38. Arnon R, Sela M. Immunomodulation by the copolymer glatiramer acetate. Journal of Molecular Recognition. 2003. pp. 412-421. doi:10.1002/jmr.628

39. Aharoni R, Teitelbaum D, Sela M, Arnon R. Copolymer 1 induces T cells of the T helper type 2 that crossreact with myelin basic protein and suppress experimental autoimmune encephalomyelitis. Proceedings of the National Academy of Sciences. 1997. pp. 10821-10826. doi:10.1073/pnas.94.20.10821

40. Duda PW, Schmied MC, Cook SL, Krieger JI, Hafler DA. Glatiramer acetate (Copaxone) induces degenerate, Th2-polarized immune responses in patients with multiple sclerosis. J Clin Invest. 2000;105: 967-976.

41. Aharoni R, Kayhan B, Eilam R, Sela M, Arnon R. Glatiramer acetate-specific T cells in the brain 


\section{Running Title: GA reduces ER \& mitochondrial stress in EAE}

express T helper $2 / 3$ cytokines and brain-derived neurotrophic factor in situ. Proceedings of the National Academy of Sciences. 2003. pp. 14157-14162. doi:10.1073/pnas.2336171100

42. Aharoni R, Herschkovitz A, Eilam R, Blumberg-Hazan M, Sela M, Bruck W, et al. Demyelination arrest and remyelination induced by glatiramer acetate treatment of experimental autoimmune encephalomyelitis. Proc Natl Acad Sci U S A. 2008;105: 11358-11363.

43. Nishitoh H. CHOP is a multifunctional transcription factor in the ER stress response. Journal of biochemistry. 2012. pp. 217-219.

44. Liu Z, Lv Y, Zhao N, Guan G, Wang J. Protein kinase R-like ER kinase and its role in endoplasmic reticulum stress-decided cell fate. Cell Death Dis. 2015;6: e1822.

45. Narendra DP, Jin SM, Tanaka A, Suen D-F, Gautier CA, Shen J, et al. PINK1 is selectively stabilized on impaired mitochondria to activate Parkin. PLoS Biol. 2010;8: e1000298.

46. Kong X, Wang R, Xue Y, Liu X, Zhang H, Chen Y, et al. Sirtuin 3, a New Target of PGC-1 $\alpha$, Plays an Important Role in the Suppression of ROS and Mitochondrial Biogenesis. PLoS ONE. 2010. p. e11707. doi:10.1371/journal.pone.0011707

47. Chen H, Detmer SA, Ewald AJ, Griffin EE, Fraser SE, Chan DC. Mitofusins Mfn1 and Mfn2 coordinately regulate mitochondrial fusion and are essential for embryonic development. J Cell Biol. 2003;160: 189-200.

48. Mai S, Klinkenberg M, Auburger G, Bereiter-Hahn J, Jendrach M. Decreased expression of Drp1 and Fis 1 mediates mitochondrial elongation in senescent cells and enhances resistance to oxidative stress through PINK1. J Cell Sci. 2010;123: 917-926.

49. Cerveny KL, McCaffery JM, Jensen RE. Division of mitochondria requires a novel DNM1interacting protein, Net2p. Mol Biol Cell. 2001;12: 309-321.

50. Deslauriers AM, Afkhami-Goli A, Paul AM, Bhat RK, Acharjee S, Ellestad KK, et al. Neuroinflammation and Endoplasmic Reticulum Stress Are Coregulated by Crocin To Prevent Demyelination and Neurodegeneration. The Journal of Immunology. 2011. pp. 4788-4799. doi:10.4049/jimmunol.1004111

51. Haile Y, Deng X, Ortiz-Sandoval C, Tahbaz N, Janowicz A, Lu J-Q, et al. Rab32 connects ER stress to mitochondrial defects in multiple sclerosis. J Neuroinflammation. 2017;14: 19.

52. Yang H, Yang T, Baur JA, Perez E, Matsui T, Carmona JJ, et al. Nutrient-sensitive mitochondrial NAD+ levels dictate cell survival. Cell. 2007;130: 1095-1107.

53. Garten A, Schuster S, Penke M, Gorski T, de Giorgis T, Kiess W. Physiological and pathophysiological roles of NAMPT and NAD metabolism. Nature Reviews Endocrinology. 2015. pp. 535-546. doi:10.1038/nrendo.2015.117

54. Li X, Kazgan N. Mammalian sirtuins and energy metabolism. Int J Biol Sci. 2011;7: 575-587.

55. Zhang X, Tang N, Hadden TJ, Rishi AK. Akt, FoxO and regulation of apoptosis. Biochim Biophys Acta. 2011;1813: 1978-1986.

56. Westphal D, Kluck RM, Dewson G. Building blocks of the apoptotic pore: how Bax and Bak are activated and oligomerize during apoptosis. Cell Death Differ. 2014;21: 196-205. 


\section{Running Title: GA reduces ER \& mitochondrial stress in EAE}

57. Garrido C, Galluzzi L, Brunet M, Puig PE, Didelot C, Kroemer G. Mechanisms of cytochrome c release from mitochondria. Cell Death Differ. 2006;13: 1423-1433.

58. Weber MS, Hohlfeld R, Zamvil SS. Mechanism of action of glatiramer acetate in treatment of multiple sclerosis. Neurotherapeutics. 2007;4: 647.

59. Filippi M, Rovaris M, Rocca MA, Sormani MP, Wolinsky JS, Comi G, et al. Glatiramer acetate reduces the proportion of new MS lesions evolving into "black holes." Neurology. 2001;57: 731733.

60. De Kleijn KMA, Martens GJM. Molecular Effects of FDA-Approved Multiple Sclerosis Drugs on Glial Cells and Neurons of the Central Nervous System. Int J Mol Sci. 2020;21. doi:10.3390/ijms21124229

61. Kadowaki H, Nishitoh H. Signaling Pathways from the Endoplasmic Reticulum and Their Roles in Disease. Genes. 2013. pp. 306-333. doi:10.3390/genes4030306

62. Li A, Song N-J, Riesenberg BP, Li Z. The Emerging Roles of Endoplasmic Reticulum Stress in Balancing Immunity and Tolerance in Health and Diseases: Mechanisms and Opportunities. Front Immunol. 2019;10: 3154.

63. Zhang K, Kaufman RJ. From endoplasmic-reticulum stress to the inflammatory response. Nature. 2008. pp. 455-462. doi:10.1038/nature 07203

64. Andhavarapu S, Mubariz F, Arvas M, Bever C Jr, Makar TK. Interplay between ER stress and autophagy: A possible mechanism in multiple sclerosis pathology. Exp Mol Pathol. 2019;108: 183190.

65. Lin W, Popko B. Endoplasmic reticulum stress in disorders of myelinating cells. Nat Neurosci. 2009;12: 379-385.

66. Meares GP, Liu Y, Rajbhandari R, Qin H, Nozell SE, Mobley JA, et al. PERK-dependent activation of JAK1 and STAT3 contributes to endoplasmic reticulum stress-induced inflammation. Mol Cell Biol. 2014;34: 3911-3925.

67. Ní Fhlathartaigh M, McMahon J, Reynolds R, Connolly D, Higgins E, Counihan T, et al. Calreticulin and other components of endoplasmic reticulum stress in rat and human inflammatory demyelination. Acta Neuropathol Commun. 2013;1: 37.

68. Lin W, Harding HP, Ron D, Popko B. Endoplasmic reticulum stress modulates the response of myelinating oligodendrocytes to the immune cytokine interferon- $\gamma$. J Cell Biol. 2005;169: 603.

69. Sarrabeth Stone WL. The unfolded protein response in multiple sclerosis. Front Neurosci. 2015;9. doi:10.3389/fnins.2015.00264

70. Lin W, Kunkler PE, Harding HP, Ron D, Kraig RP, Popko B. Enhanced integrated stress response promotes myelinating oligodendrocyte survival in response to interferon-gamma. Am J Pathol. 2008;173: 1508-1517.

71. Way SW, Podojil JR, Clayton BL, Zaremba A, Collins TL, Kunjamma RB, et al. Pharmaceutical integrated stress response enhancement protects oligodendrocytes and provides a potential multiple sclerosis therapeutic. Nat Commun. 2015;6: 6532. 


\section{Running Title: GA reduces ER \& mitochondrial stress in EAE}

72. Guthrie LN, Abiraman K, Plyler ES, Sprenkle NT, Gibson SA, McFarland BC, et al. Attenuation of PKR-like ER Kinase (PERK) Signaling Selectively Controls Endoplasmic Reticulum Stress-induced Inflammation Without Compromising Immunological Responses. J Biol Chem. 2016;291: 15830 15840.

73. Deng H, Dodson MW, Huang H, Guo M. The Parkinson's disease genes pink1 and parkin promote mitochondrial fission and/or inhibit fusion in Drosophila. Proceedings of the National Academy of Sciences. 2008. pp. 14503-14508. doi:10.1073/pnas.0803998105

74. Poole AC, Thomas RE, Andrews LA, McBride HM, Whitworth AJ, Pallanck LJ. The PINK1/Parkin pathway regulates mitochondrial morphology. Proc Natl Acad Sci U S A. 2008;105: 1638-1643.

75. Calì T, Ottolini D, Negro A, Brini M. Enhanced parkin levels favor ER-mitochondria crosstalk and guarantee $\mathrm{Ca}(2+)$ transfer to sustain cell bioenergetics. Biochim Biophys Acta. 2013;1832: 495-508.

76. Parkinson's disease-associated parkin colocalizes with Alzheimer's disease and multiple sclerosis brain lesions. Neurobiol Dis. 2009;36: 445-452.

77. Lieberknecht V, Junqueira SC, Cunha MP, Barbosa TA, de Souza LF, Coelho IS, et al. Pramipexole, a Dopamine D2/D3 Receptor-Preferring Agonist, Prevents Experimental Autoimmune Encephalomyelitis Development in Mice. Mol Neurobiol. 2016;54: 1033-1045.

78. Buhlman L, Damiano M, Bertolin G, Ferrando-Miguel R, Lombès A, Brice A, et al. Functional interplay between Parkin and Drp1 in mitochondrial fission and clearance. Biochim Biophys Acta. 2014;1843. doi:10.1016/j.bbamcr.2014.05.012

79. Ham SJ, Lee D, Yoo H, Jun K, Shin H, Chung J. Decision between mitophagy and apoptosis by Parkin via VDAC1 ubiquitination. Proc Natl Acad Sci U S A. 2020;117: 4281-4291.

80. Muñoz JP, Ivanova S, Sánchez-Wandelmer J, Martínez-Cristóbal P, Noguera E, Sancho A, et al. Mfn2 modulates the UPR and mitochondrial function via repression of PERK. EMBO J. 2013;32: $2348-2361$.

81. McLelland G-L, Goiran T, Yi W, Dorval G, Chen CX, Lauinger ND, et al. Mfn2 ubiquitination by PINK1/parkin gates the p97-dependent release of ER from mitochondria to drive mitophagy. Elife. 2018;7. doi:10.7554/eLife.32866

82. van Vliet AR, Verfaillie T, Agostinis P. New functions of mitochondria associated membranes in cellular signaling. Biochimica et Biophysica Acta (BBA) - Molecular Cell Research. 2014. pp. 2253-2262. doi:10.1016/j.bbamcr.2014.03.009

83. Kim H, Scimia MC, Wilkinson D, Trelles RD, Wood MR, Bowtell D, et al. Fine-tuning of Drp1/Fis1 availability by AKAP121/Siah2 regulates mitochondrial adaptation to hypoxia. Mol Cell. 2011;44: $532-544$.

84. Luo F, Herrup K, Qi X, Yang Y. Inhibition of Drp1 hyper-activation is protective in animal models of experimental multiple sclerosis. Exp Neurol. 2017;292: 21.

85. Shindler KS, Ventura E, Dutt M, Elliott P, Fitzgerald DC, Rostami A. Oral Resveratrol Reduces Neuronal Damage in a Model of Multiple Sclerosis. Journal of Neuro-Ophthalmology. 2010. pp. 328-339. doi:10.1097/wno.0b013e3181f7f833

86. Giralt A, Hondares E, Villena JA, Ribas F, Díaz-Delfín J, Giralt M, et al. Peroxisome Proliferator- 


\section{Running Title: GA reduces ER \& mitochondrial stress in EAE}

activated Receptor- $\gamma$ Coactivator- $1 \alpha$ Controls Transcription of theSirt3Gene, an Essential Component of the Thermogenic Brown Adipocyte Phenotype. Journal of Biological Chemistry.

2011. pp. 16958-16966. doi:10.1074/jbc.m110.202390

87. Tegla CA, Azimzadeh P, Andrian-Albescu M, Martin A, Cudrici CD, Trippe R 3rd, et al. SIRT1 is decreased during relapses in patients with multiple sclerosis. Exp Mol Pathol. 2014;96: 139-148.

88. Hewes D, Tatomir A, Kruszewski AM, Rao G, Tegla CA, Ciriello J, et al. SIRT1 as a potential biomarker of response to treatment with glatiramer acetate in multiple sclerosis. Exp Mol Pathol. 2017;102: 191-197.

89. Rice C, Gray E, Hares K, Kemp K, Sun M, Ginty M, et al. SIRT3 expression is reduced in nonlesional grey matter in multiple sclerosis. 2013 [cited 28 Oct 2020]. Available:

https://www.semanticscholar.org/paper/SIRT3-expression-is-reduced-in-non-lesional-grey-in-RiceGray/74a016f59adf3bc519a49078255220066a86f248

90. Yu R, Lendahl U, Nistér M, Zhao J. Regulation of Mammalian Mitochondrial Dynamics: Opportunities and Challenges. Front Endocrinol . 2020;11. doi:10.3389/fendo.2020.00374 